\title{
The involvement of microRNA in the pathogenesis of Richter syndrome
}

Haematologica 2019

Volume 104(5):1004-1015

\section{Correspondence: \\ GEORGE A. CALIN \\ gcalin@mdanderson.org \\ Received: August 8, 2018. \\ Accepted: November 8, 2018. \\ Pre-published: November 8, 2018.}

doi:10.3324/haematol.2018.203828

Check the online version for the most updated information on this article, online supplements, and information on authorship \& disclosures: www.haematologica.org/content/104/5/1004

(C)2019 Ferrata Storti Foundation

Material published in Haematologica is covered by copyright. All rights are reserved to the Ferrata Storti Foundation. Use of published material is allowed under the following terms and conditions:

https://creativecommons.org/licenses/by-nc/4.0/legalcode. Copies of published material are allowed for personal or internal use. Sharing published material for non-commercial purposes is subject to the following conditions:

https://creativecommons.org/licenses/by-nc/4.0/legalcode, sect. 3. Reproducing and sharing published material for commercial purposes is not allowed without permission in writing from the publisher.

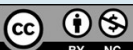

\author{
Katrien Van Roosbroeck, ${ }^{1,2 *}$ Recep Bayraktar, ${ }^{1 *}$ Steliana Calin, ${ }^{3}$ Johannes \\ Bloehdorn, ${ }^{4}$ Minnea Paul Dragomir, ${ }^{1}$ Keishi Okubo, ${ }^{1}$ Maria Teresa Sabrina \\ Bertilaccio, ${ }^{1}$ Simonetta Zupo, ${ }^{5}$ M. James You, ${ }^{3}$ Gianluca Gaidano, ${ }^{6}$ Davide \\ Rossi, ${ }^{7}$ Shih-Shih Chen, ${ }^{8}$ Nicholas Chiorazzi, ${ }^{8}$ Philip A. Thompson, ${ }^{9}$ Alessandra \\ Ferrajoli, ${ }^{9}$ Francesco Bertoni, ${ }^{7}$ Stephan Stilgenbauer, ${ }^{4}$ Michael J. Keating ${ }^{9}$ and \\ George A. Calin ${ }^{1,9,10}$
}

${ }^{1}$ Department of Experimental Therapeutics, The University of Texas MD Anderson Cancer Center, Houston, TX, USA; ${ }^{2}$ Present address - Department of Investigational Cancer Therapeutics, The University of Texas MD Anderson Cancer Center, Houston, TX, USA; ${ }^{3}$ Department of Hematopathology, The University of Texas MD Anderson Cancer Center, Houston, TX, USA; ${ }^{4}$ Department of Internal Medicine III, University Hospital UIm, Germany; ${ }^{5}$ Molecular Diagnostic Laboratory, Pathology Department, IRCCS, Ospedale Policlinico San Martino, Genoa, Italy; ${ }^{6}$ Division of Hematology, Department of Translational Medicine, University of Eastern Piedmont, Novara, Italy; ${ }^{7}$ Università della Svizzera italiana, Institute of Oncology Research, Bellinzona, Switzerland; ${ }^{8}$ The Feinstein Institute for Medical Research, Northwell Health, Manhasset, NY, USA; 'Department of Leukemia, The University of Texas MD Anderson Cancer Center, Houston, TX, USA and ${ }^{10} \mathrm{Center}$ for RNA Interference and Non-Coding RNAs, The University of Texas MD Anderson Cancer Center, Houston, TX, USA

${ }^{\star} K V R$ and $R B$ contibuted equally to this work.

\section{ABSTRACT}

$\mathrm{R}$ ichter syndrome is the name given to the transformation of the most frequent type of leukemia, chronic lymphocytic leukemia, into an aggressive lymphoma. Patients with Richter syndrome have limited response to therapies and dismal survival. The underlying mechanisms of transformation are insufficiently understood and there is a major lack of knowledge regarding the roles of microRNA that have already proven to be causative for most cases of chronic lymphocytic leukemia. Here, by using four types of genomic platforms and independent sets of patients from three institutions, we identified microRNA involved in the transformation of chronic lymphocytic leukemia to Richter syndrome. The expression signature is composed of miR-21, miR-150, miR-146b and miR-181b, with confirmed targets significantly enriched in pathways involved in cancer, immunity and inflammation. In addition, we demonstrated that genomic alterations may account for microRNA deregulation in a subset of cases of Richter syndrome. Furthermore, network analysis showed that Richter transformation leads to a complete rearrangement, resulting in a highly connected microRNA network. Functionally, ectopic overexpression of miR-21 increased proliferation of malignant $B$ cells in multiple assays, while miR-150 and miR26 a were downregulated in a chronic lymphocytic leukemia xenogeneic mouse transplantation model. Together, our results suggest that Richter transformation is associated with significant expression and genomic loci alterations of microRNA involved in both malignancy and immunity.

\section{Introduction}

The most frequent type of adult leukemia, chronic lymphocytic leukemia (CLL), is a disease in which alterations of small non-coding RNA named microRNA (miRNA, miR) play a fundamental role: the miR-15a/16-1 cluster at the $13 \mathrm{q}$ deletion hotspot, which targets the oncogenic anti-apoptotic proteins BCL2 and MCL1, is deleted or downregulated in most and germline-mutated in some patients. ${ }^{1-3}$ Although these discoveries were made more than a decade ago 
as a first link between non-coding RNA alterations and human diseases, ${ }^{4,5}$ the mechanistic involvement of miRNA in the CLL patients with the worst prognosis, those whose disease transforms to Richter syndrome (RS), has not been reported to date. RS occurs in up to $8 \%$ of untreated CLL patients ${ }^{6}$ and in $5-16 \%$ of patients treated with targeted therapies, such as ibrutinib or venetoclax for relapsed CLL.,8 Abnormalities of regulators of tumor suppression (TP53), cell proliferation (NOTCH1, MYC) and cell cycle (CDKN2A), have been reported in RS, ${ }^{9}$ but biomarkers to predict the occurrence of RS are lacking at present. RS is characterized by rapid progression and outcomes of patients treated with a variety of moderate or high-intensity chemoimmunotherapy regimens are uniformly dismal, with a median survival of less than 1 year, ${ }^{10-13}$ particularly for patients with clonally-related or TP53-mutated disease. ${ }^{14}$ Novel, molecularly targeted approaches are urgently required, but this is hampered by the limited understanding of the molecular pathogenesis of RS. The paucity of molecular studies is mainly due to the scarceness of biopsy materials. Furthermore, the availability of non-invasive methods of diagnosis (such as the use of 2deoxy-2-[(18)F] fluoroglucose/positron emission tomography, ${ }^{15}$ reduces the need for follow-up biopsies, which further limits the availability of material for research. Therefore, there is a strong need to develop RS biomarkers and molecularly targeted therapies that could facilitate early and accurate diagnosis, as well as assist current treatment strategies. In the present study, we investigated the expression and potential roles of miRNA in the transformation from CLL to RS, as these miRNA could be therapeutically targeted.

\section{Methods}

\section{Patients' samples \\ The University of Texas MD Anderson Cancer Center (UTMDACC) cohort}

The "paired" set: 14 bone marrow samples from seven patients with RS were collected at the UTMDACC. For each patient, samples from the time of CLL diagnosis (group 1a) and Richter transformation (group 1b) were available. In addition, we collected 14 bone marrow samples from seven age-, sexand sample time-matched CLL control patients who did not develop RS over the course of follow-up at the UTMDACC. For each patient, a sample at the time of CLL diagnosis (group $2 \mathrm{a})$ and at a time corresponding to the time of RS diagnosis of group 1 (group 2b) were available. Online Supplementary Table $S 1$ shows that age at diagnosis, gender and time to transformation were not significantly different between patients of this paired RS/CLL cohort.

The "extended" set : we also extended our initial paired RS/CLL cohort to include samples from 27 patients with RS [25 samples at CLL diagnosis (group 1a) and 9 samples at the time of Richter transformation (group 1b)] and 23 control CLL patients [17 samples at CLL diagnosis (group 2a) and 14 samples at a time corresponding to the time of Richter transformation in the RS group (group 2b)]. All samples used were formalin-fixed paraffin-embedded (FFPE) bone marrow cores, except for one lymph node sample in group $1 \mathrm{~b}$. A schematic representation of the extended cohort is shown in Figure 1A,B, while the patients' characteristics are presented in Table 1 and detailed in Online Supplementary Table S2.

\section{UIm University cohort}

We used peripheral blood from 58 fludarabine-resistant patients. Samples were taken at enrollment before treatment. Eight of these 58 patients subsequently developed RS. These patients were described previously. ${ }^{16}$

\section{The Bellinzona Institute of Oncology Research cohort}

This cohort of patients comprised 737 cases of mature lymphoid tumors including CLL, and were described previously. ${ }^{17}$

The study was approved by the institutional review boards and ethical committees of all the participating institutions.

\section{Xenogeneic mouse transplantation}

We used a previously described mouse model of CLL for in vivo experiments. ${ }^{18-20}$

\section{Firefly microRNA profiling assay}

We performed expression analysis of 40 human and viral miRNA known to be involved in the progression of CLL, associated with poor prognosis CLL, highly expressed in CLL as determined by a previously performed RNA-sequencing study, ${ }^{21}$ located in genomic regions reported to be deregulated in $\mathrm{RS},{ }^{17}$ or frequently reported in literature to be associated with CLL (Online Supplementary Table S3). We used a Firefly custom multiplex circulating miRNA assay (Abcam, Cambridge, MA, USA) for the extended cohort of CLL/RS samples collected at the UTMDACC, as described in the Online Supplementary Methods.

\section{Polymerase chain reaction analysis and microRNA gene expression profiling}

Quantitative reverse transcription polymerase chain reaction (qRT-PCR) and miRNA gene expression profiling for miRNA profiling confirmation are described in detail in the Online Supplementary Methods.

\section{Genome-wide DNA profiles}

Genome-wide DNA profiles were obtained from high-molecular-weight genomic DNA using the Genome-Wide Human SNP Array 6.0 (Affymetrix, Santa Clara, CA, USA), as previously reported (GSE50252). ${ }^{17}$

\section{Network analyses}

The miRNA networks were generated as previously described; ${ }^{22}$ the method is detailed in the Online Supplementary Methods.

\section{Results}

\section{A microRNA signature is involved in the process of Richter transformation}

As illustrated in the workflow in Figure 1A, we first designed the UTMDACC cohort consisting of patients with RS and age-, sex- and sample time-matched CLL "controls". For all of these cases FFPE bone marrow samples, taken at the time of CLL diagnosis and at the time of RS diagnosis, or a time corresponding to the RS diagnosis in the case of the matched CLL controls, were available and analyzed (Figure $1 \mathrm{~B}$ and Online Supplementary Table S1). We decided to use the Firefly custom multiplex miRNA assay due to the best data generation/cost ratio, and well annotated and selected miRNA (see Methods). We identified nine miRNA potentially involved in the RS transformation process, 
i.e. significantly differentially expressed (SDE, at $P$ values $<0.05$ ) in bone marrow samples of patients with CLL at the time of Richter transformation when compared to their expression in samples obtained at the time of CLL diagnosis: SDE in group $1 \mathrm{~b}$ versus group $1 \mathrm{a}$, but not in group $2 b$ versus group $2 a$ (to exclude the time of evolution as a variable) (Figures $1 \mathrm{C}$ and 2A,B, Online Supplementary Figure $S 1$ and Online Supplementary Table S2). We excluded miR-34a and miR-17 from the list of Richter-specific SDE miRNA, as these were also significantly upregulated in the control group of patients with CLL who did not develop RS over the course of followup, which was similar to the time to Richter transformation in the RS group. We then extended our cohort to include non-matched samples (Table 1 and Online Supplementary Table S2) and analyzed these with the Firefly custom multiplex miRNA assay as well. We considered the following groups (Figure 1B): group 1a - samples at the time of CLL diagnosis from patients with
CLL who later developed RS ( $n=25)$; group $1 b$ - samples from patients with CLL at the time of Richter transformation/RS diagnosis ( $\mathrm{n}=9)$; group $2 \mathrm{a}$ - control samples from patients with CLL, at the time of diagnosis, who did not develop RS over a follow-up period equal to or longer than the time between CLL and RS diagnosis of groups $1 \mathrm{a}$ and $1 \mathrm{~b},(\mathrm{n}=16)$; and group $2 \mathrm{~b}$ - at a time corresponding to RS transformation in group $1 b \quad(n=13)$. This analysis revealed 15 miRNA that showed significantly different expression between samples at the time of CLL diagnosis (group 1a) and at the time of RS diagnosis (group $1 b$ ), of which seven are common with the paired analysis (Figure 2A-C and Online Supplementary Figure S2).

Next, we validated these results with a different assay and performed qRT-PCR expression analysis on 25 miRNA in the same extended CLL/RS dataset. This analysis confirmed three differentially expressed miRNA: miR-21 and miR-146b were upregulated and

A

\section{Step 1: miRNA profiling}

- Firelfy targeted profiling of 40 miRNAs in a paired set of CLL to RT samples from MDACC

- Firefly targeted profiling of 40 miRNAs in an extended set of CLL to RT samples from MDACC

- qRT-PCR confirmation in the extended set of CLL to RT samples from MDACC

- Confirmation in an independent set of CLL vs. RS samples from Ulm University

\section{Step 2: aCGH analysis}

- Analysis of an aCGH dataset of 15 paired cases (CLL phase/RT phase) from IOR Belinzona - Analysis of an aCGH dataset of 737 cases of mature lymphoid tumors from IOR Belinzona

\section{Step 3: Functional analysis}

- Pathway analysis of confirmed miRNA targets

- miRNA expression network analysis

- Functional studies in CLL/DLBCL cell lines

- miRNA analysis after transfer of CLL derived B-cells into NSG mice

B Patients with
Richter

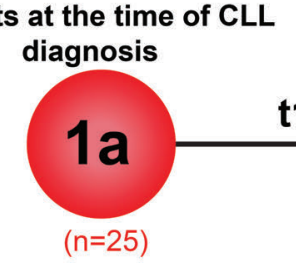

Patients at the time of CLL diagnosis

\section{Patients with} CLL

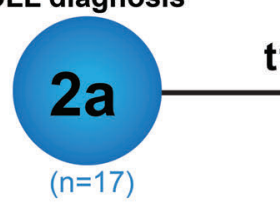

Patients at the time of Richter transformation

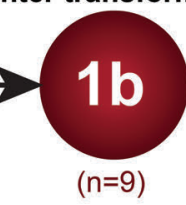

CLL patients at the same follow-up time as RS

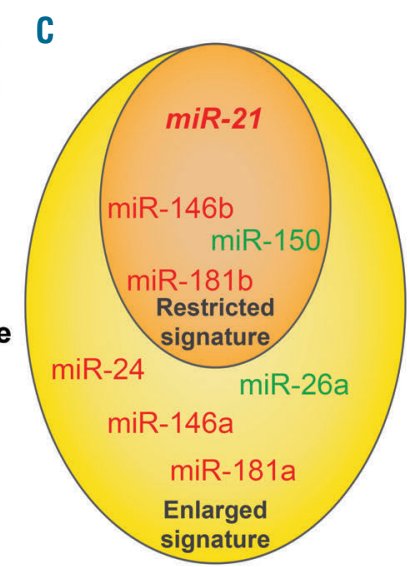

$1 b$ vs $1 a$, but not in $2 b$ vs $2 a$

Figure 1. Workflow of the microRNA screening process and composition of the University of Texas MD Anderson Cancer Center cohort of patients with Richter syndrome/chronic lymphocytic leukemia. (A) Three-step workflow of the miRNA screening process, consisting of miRNA profiling, array comparative genomic hybridization $(\mathrm{aCGH})$ analysis and functional analysis. (B) Schematic representation of the extended University of Texas MD Anderson Cancer Center (MDACC) cohort of patients. This cohort consists of patients with Richter syndrome (RS) (group 1), as well as age-, sex- and sample time-matched controls with chronic lymphocytic leukemia (CLL) (group 2). For both groups, samples at CLL diagnosis (group 1a) and at the time of RS diagnosis (group 1b) or a time corresponding to RS diagnosis time in the case of the matched CLL controls (group 2b) are available. Time "t1" between CLL diagnosis and RS diagnosis time is similar for both groups. (C) miR-21, miR-146a, miR-150 and miR-181b are members of the four-miRNA "restricted signature", while the eight-miRNA "enlarged signature" contains these four miRNA and additionally miR-24, miR-26a, miR-181a and miR-146a. miRNA highlighted in red are upregulated in RS, while miRNA highlighted in green are downregulated. For additional Information, see Online Supplementary Figure S1. IOR: Institute of Oncology Research; DLBCL: diffuse large B-cell lymphoma. 
miR-150 was downregulated at the time of Richter transformation (Figures 2A and 3A). Finally, to further confirm our findings, we analyzed the levels of the differentially expressed human miRNA in an independent set of 58 patients with CLL/RS from Ulm University ${ }^{16}$ for whom microarray data were available (Online Supplementary Table S4). We confirmed that three miRNA, miR-21, miR-146b and miR-181b, were all upregulated in RS when compared to CLL (Figures $2 \mathrm{~A}$ and 3B,C). Taken together, miR-21, a well-known oncogene, ${ }^{23}$ was found by all four analyses to be significantly more highly expressed at the time of Richter transformation than at the time of CLL diagnosis, strongly suggesting a role of this miRNA in the process of transformation to RS. In addition, three miRNA were found to be differentially expressed by three analyses: miR-146b and miR-181b were upregulated and miR-150 was downregulated at the time of Richter transformation. We will refer to this signature of four miRNA as the "restricted signature". In addition, four miRNA were significantly different in two analyses: miR-26a was significantly downregulated, while miR-24, miR-146a and miR-181a were significantly upregulated at the time of RS diagnosis. This signature of eight miRNA was designated as the "enlarged signature" (Figures 1C and 2A). Of note, miRNA from the same families - miR-181a and miR-181b, and miR-146a and miR-146b - were present in both signatures. Three viral miRNA, the Epstein-Barr virus miRNA BART4 and BART16, and the Kaposi sarcoma herpes virus miRNA kshv-miR-K12-4 were differentially expressed in the Firefly analysis of all samples, but could not be evaluated in a cohort from an independent institution, as the array used to investigate the Ulm University cohort did not contain probes for non-human miRNA.

\section{Genomic alterations may account for microRNA deregulation in a subset of cases of Richter syndrome}

miRNA are often located at fragile sites in human and mouse genomes. ${ }^{24,25}$ To investigate whether the differen-
A
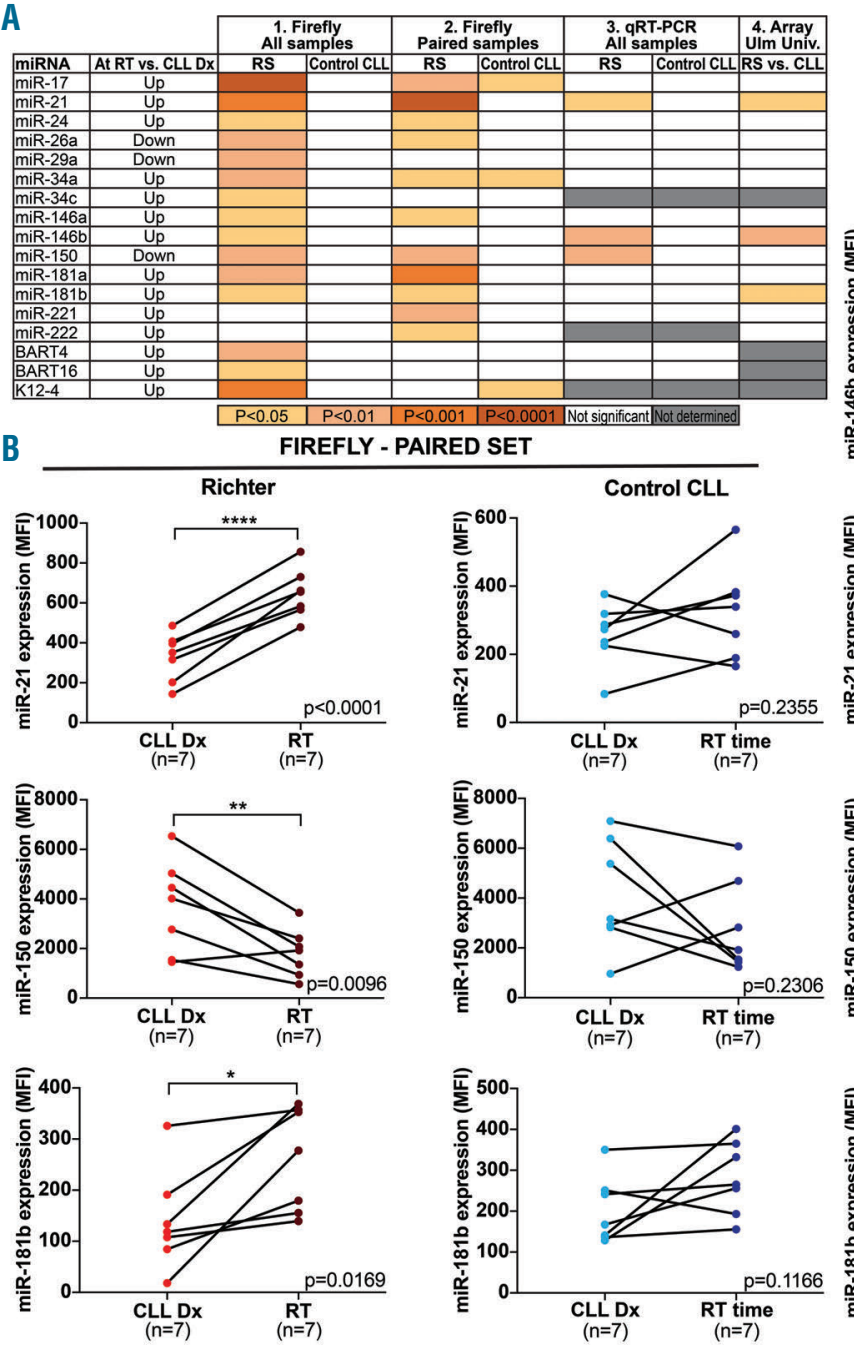
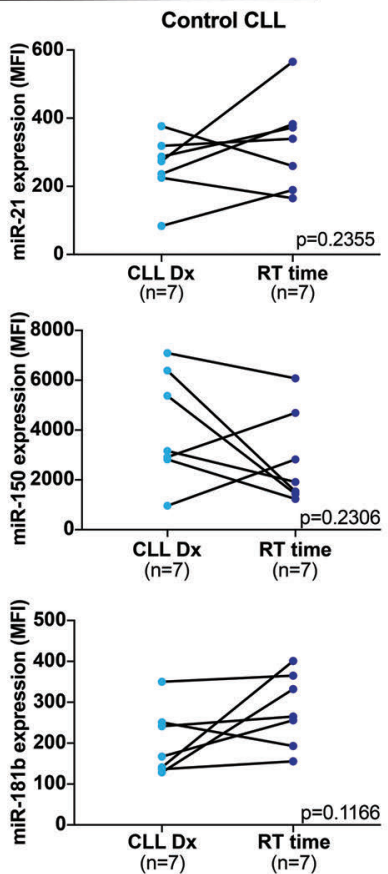

c
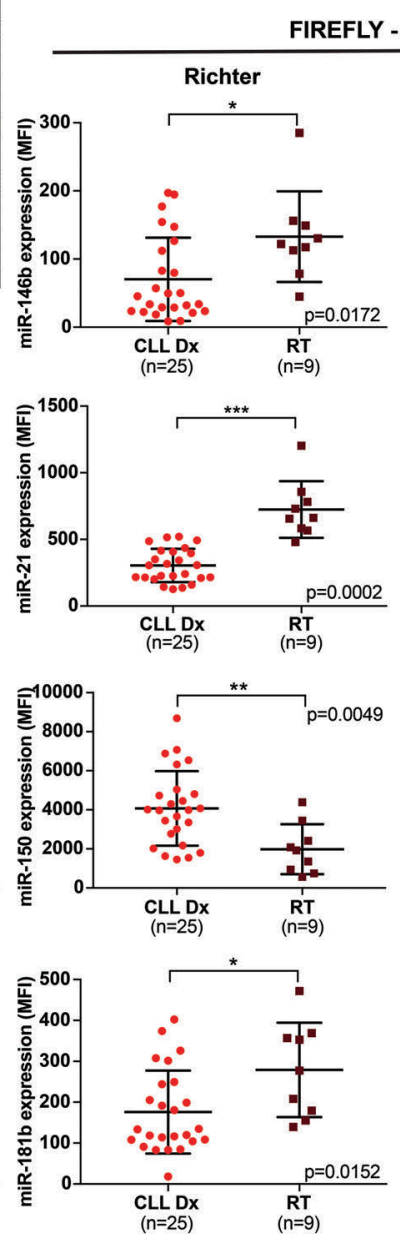
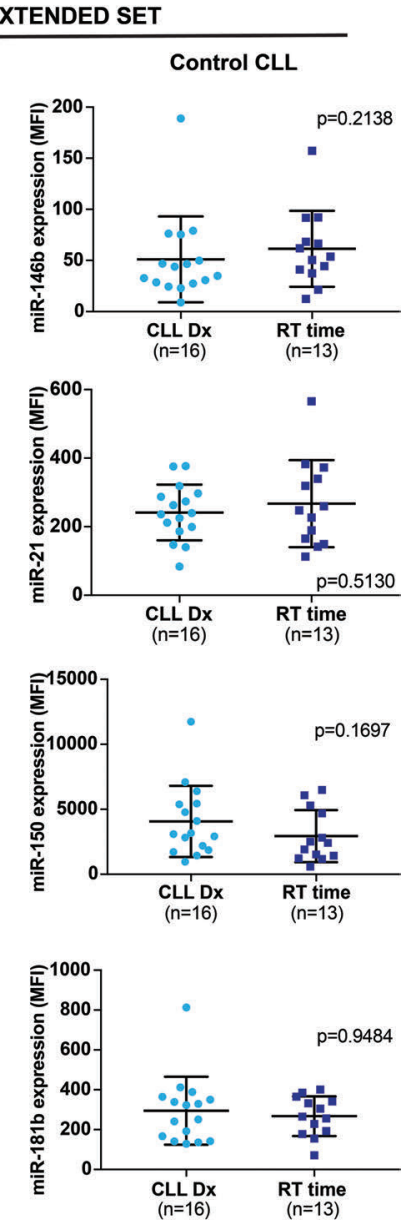

Figure 2. microRNA profiling with the Firefly custom multiplex microRNA assay. (A) Summary of the significantly expressed miRNA in the profiling step of the project. (B) miR-21 and miR-181b are significantly upregulated, and miR-150 is significantly downregulated at the time of Richter transformation (RT) when compared to at chronic lymphocytic leukemia (CLL) diagnosis (Dx) for the paired Richter syndrome (RS) samples, but not for the paired control CLL samples. (C) miR-146b, miR-21 and miR-181b are significantly upregulated, and miR-150 is significantly downregulated at RT time when compared to at CLL diagnosis in the extended set of RS samples, but not in the extended set of control CLL samples. * $P<0.05 ; * * P<0.01 ; * * * P<0.001$. For additional Information, see Online Supplementary Figure S2. 
tially expressed human miRNA are located in regions genetically altered in RS, we first analyzed the eight genomic loci of the enlarged signature-related miRNA in 15 paired CLL/RS cases for which samples at CLL diagnosis and at RS diagnosis were available. ${ }^{17}$ We found that, in RS samples, three cases $(20 \%)$ had a gain of the miR$181 \mathrm{a} / \mathrm{b}$ clusters on either chromosome 1q32 or 9q33, three cases (20\%) had a loss of miR-26a-1 and a-2 loci on either chromosome $3 \mathrm{p} 22$ or $12 \mathrm{q} 14$, two cases $(13.5 \%)$ had a gain of miR-21 locus at 17q23, and one case $(6.67 \%)$ had a loss of miR-150 locus at 19q13 (Figure 4A). We then considered a larger series of cases of RS $(n=59)$ and CLL phases $(\mathrm{n}=28)$. In accordance with the abovepresented expression data, we observed a similar pattern of miRNA-specific genetic aberrations: RS patients more frequently presented gains affecting miR-21, miR-146a and miR-181a/b genomic loci (Online Supplementary Figure S4A). Finally, we took advantage of a series of 737 genomic profiles obtained in mature lymphoid tumors to compare the frequency of DNA copy number aberrations with that observed in RS. ${ }^{17,26-29}$ The same miRNA loci were sites of gains $(\mathrm{miR}-181 \mathrm{a} / \mathrm{b}, 6 \%$ and $8 \%$ at $1 \mathrm{q}$ or $9 \mathrm{q}$, respectively; miR-21, 4\%; miR-146a, 3\%; miR-146b, $0.2 \%$ ) or losses (miR-150, $6 \%$; miR-26a, $3 \%$ and $0.1 \%$ at $3 p$ or $12 q$, respectively) in mature lymphoid tumors (Figure 4B and Online Supplementary Figure S4B), but at a significantly lower frequency than that observed in RS samples $\left(P=0.017\right.$ and $\chi^{2}=5.669$, see Online Supplementary Figure S4C). Altogether, these data suggest that, at least in some cases, Richter-related miRNA are deregulated due to DNA copy number changes, which occur during the transformation process, and are enriched with respect to non-Richter B-cell malignancies. The mechanism(s) for the miRNA expression deregulation for the majority of Richter cases has still to be identified.

\section{The microRNA network is reprogrammed during Richter transformation}

Although it is known that changes in miRNA expression are involved in the initiation and development of CLL, ${ }^{30}$ we further investigated whether analysis of the miRNA interactor network could add more information

Table 1. Characteristics of patients with Richter syndrome and chronic lymphocytic leukemia in the cohort from the University of Texas MD Anderson Cancer Center.

\begin{tabular}{|c|c|c|c|c|c|}
\hline Characteristic & Rich & 27) & & 23) & P-value \\
\hline & N. & $\%$ & N. & $\%$ & \\
\hline Age at diagnosis, years & & & & & \\
\hline Median & 54 & & 59 & & 0.1973 \\
\hline Range & $31-78$ & & $43-70$ & & \\
\hline Sex & & & & & \\
\hline Male & 17 & 63.0 & 14 & 60.9 & $>0.9999$ \\
\hline Female & 10 & 37.0 & 9 & 39.1 & \\
\hline Rai stage & & & & & \\
\hline 0 & 4 & 14.8 & 7 & 30.5 & 0.0947 \\
\hline 1 & 11 & 40.7 & 13 & 56.5 & \\
\hline 2 & 5 & 18.5 & 1 & 4.3 & \\
\hline 3 & 4 & 14.8 & 0 & 0.0 & \\
\hline 4 & 1 & 3.7 & 2 & 8.7 & \\
\hline NA & 2 & 7.4 & 0 & 0.0 & \\
\hline FISH & & & & & \\
\hline dell3q & 8 & 29.6 & 16 & 69.6 & 0.0328 \\
\hline NL cyto/FISH & 4 & 14.8 & 0 & 0.0 & \\
\hline trisomy 12 & 8 & 29.6 & 5 & 21.8 & \\
\hline dell1q & 6 & 22.2 & 3 & 13.1 & \\
\hline del17p & 5 & 18.5 & 1 & 4.35 & \\
\hline NA & 4 & 14.8 & 2 & 8.7 & \\
\hline ZAP70 & & & & & \\
\hline Positive & 6 & 22.2 & 8 & 34.8 & 0.0239 \\
\hline Negative & 0 & 0.0 & 10 & 43.5 & \\
\hline NA & 21 & 77.8 & 5 & 21.8 & \\
\hline CD38 & & & & & \\
\hline Positive $(>=20 \%)$ & 17 & 63.0 & 4 & 17.4 & $<0.0001$ \\
\hline Negative $(<20 \%)$ & 3 & 11.1 & 15 & 65.3 & \\
\hline NA & 7 & 25.9 & 4 & 17.4 & \\
\hline $\mathrm{B}_{2}$ microglobulin & & & & & \\
\hline Positive ( $\geq 2 \mathrm{mg} / \mathrm{mL}$ ) & 25 & 92.6 & 18 & 78.3 & 0.5718 \\
\hline Negative $(<2 \mathrm{mg} / \mathrm{mL})$ & 1 & 3.7 & 2 & 8.7 & \\
\hline NA & 1 & 3.7 & 3 & 13 & \\
\hline $\mathrm{B}_{2}$ microglobulin level & & & & & \\
\hline Median & 3.6 & & 2.4 & & 0.0051 \\
\hline Range & $1.6-10$ & & $1-4.1$ & & \\
\hline
\end{tabular}

\begin{tabular}{|c|c|c|c|c|c|}
\hline \multirow[t]{2}{*}{ Characteristic } & \multicolumn{2}{|c|}{ Richter ( $n=27$ ) } & \multicolumn{2}{|c|}{ CLL (n=23) } & \multirow[t]{2}{*}{ P-value } \\
\hline & N. & $\%$ & N. & $\%$ & \\
\hline \multicolumn{6}{|l|}{ IGHV } \\
\hline Unmutated & 18 & 66.7 & 7 & 30.45 & 0.1459 \\
\hline Mutated & 5 & 18.5 & 7 & 30.45 & \\
\hline NA & 4 & 14.8 & 9 & 39.1 & \\
\hline \multicolumn{6}{|c|}{ Time to transformation (months) } \\
\hline Median & 69 & & NA & & \\
\hline Range & 8-213 & & NA & & \\
\hline \multicolumn{6}{|l|}{ Survival } \\
\hline Dead & 22 & 81.5 & 1 & 4.35 & $<0.0001$ \\
\hline Alive at last follow-up & 5 & 18.5 & 22 & 95.7 & \\
\hline \multicolumn{6}{|l|}{ Survival time (months) } \\
\hline Median & 108 & & 96 & & 0.3821 \\
\hline Range & $20-245$ & & $15-234$ & & \\
\hline \multicolumn{6}{|l|}{ LDH at diagnosis } \\
\hline Normal (s18 UI/L) & 12 & 44.4 & 21 & 91.3 & 0.0007 \\
\hline Elevated $(>618 \mathrm{UI} / \mathrm{L})$ & 15 & 55.6 & 2 & 8.7 & \\
\hline NA & 0 & 0.0 & 0 & 0.0 & \\
\hline Median & 647 & & 513 & & 0.0079 \\
\hline Range & $303-1587$ & & $357-1175$ & & \\
\hline \multicolumn{6}{|c|}{ Time to first treatment (months) } \\
\hline Median & 10 & & 39 & & 0.0083 \\
\hline Range & $0-156$ & & $0-156$ & & \\
\hline \multicolumn{6}{|c|}{ Number of treatments (including SCT) } \\
\hline 0 & 1 & 3.7 & 4 & 17.4 & 0.0083 \\
\hline 1 & 6 & 22.2 & 13 & 56.55 & \\
\hline 2 & 5 & 18.5 & 1 & 4.35 & \\
\hline 3 & 2 & 7.4 & 1 & 4.35 & \\
\hline 4 & 6 & 22.2 & 1 & 4.35 & \\
\hline 5 & 4 & 14.8 & 0 & 0.0 & \\
\hline 6 & 3 & 11.1 & 0 & 0.0 & \\
\hline NA & 0 & 0 & 3 & 13.1 & \\
\hline
\end{tabular}

NA: not available; FISH: fluorescence in situ hybridization; LDH: lactate dehydrogenase; SCT: stem cell transplantation. 
to our scarce understanding of the transformation of CLL to RS. We investigated the miRNA regulatory networks composed of nodes and edges, where the nodes are miRNA genes, and the edges (links) are the molecular interactions (a high statistical correlation between two miRNA nodes in a given set of patients). It is important to note that this method also recognizes miRNA that are not SDE as key elements. The network analysis provides a different perspective on the role of a miRNA than the commonly used expression profiling, and the results do not always overlap. By using the qRT-PCR expression data, we generated a 25-miRNA expression network for each of the four groups of patients of the extended UTMDACC cohort (Figure 5A). We observed that the number of edges increased significantly from 29 in the group $1 \mathrm{a}$ network to 55 in the group $1 \mathrm{~b}$ network compared to the group $2 \mathrm{a}$ and group $2 \mathrm{~b}$ networks, in which only one new extra-edge appeared, with the number of edges increasing from 53 to $54 \quad(P<0.05$, $\chi^{2}=3.913$ ) (Figure $5 \mathrm{~B}$, left panel). When comparing the behavior of the miRNA nodes in patients who underwent Richter transformation, i.e., groups $1 \mathrm{a}$ and $1 \mathrm{~b}$, we observed an increase in the connectivity of nodes $(P=0.0007)$, indicating a complete reprogramming of the
miRNA network during the transformation (Figure 5B, middle panel). When we performed the same comparison for the miRNA networks of the "control" CLL patients who did not develop RS, i.e., groups $2 \mathrm{a}$ and $2 \mathrm{~b}$, we observed no significant change in the connectivity of the nodes (Figure 5B, right panel). The miRNA network of group $1 \mathrm{a}$ is a disjointed graph with many isolated nodes which, after Richter transformation (group 1b), becomes a highly-connected graph, with only two isolated miRNA (miR-23b and miR-155) (Figure 5A). The hubs (defined as the nodes with the highest connectivity, i.e., the miRNA best connected in the network) specific for Richter transformation (hubs in group $1 \mathrm{~b}$ versus group $1 \mathrm{a}$, but not in group $2 \mathrm{a}$ versus group $2 \mathrm{~b}$ ) were miR-191, miR-17 and miR-29c, which we named hubspecific (HUS) miRNA.

Additionally, these data were confirmed when we generated an independent 40-miRNA network for the four groups of patients by using the Firefly assay expression data (Online Supplementary Figure S5). Therefore, both qRTPCR-based and Firefly-based networks confirmed that Richter transformation leads to a complete rearrangement of the miRNA network, with a significant increase in the number of edges and the addition of new miRNA hubs.
A
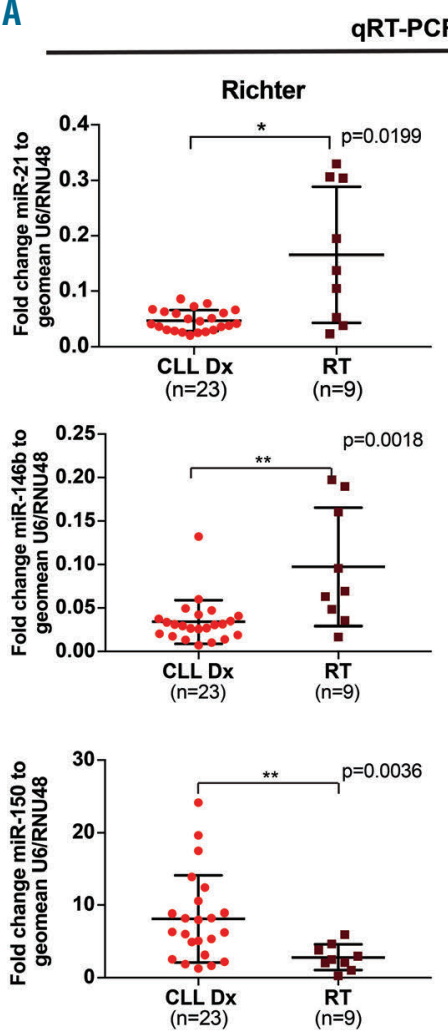

B

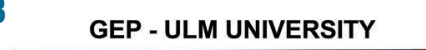

Control CLL
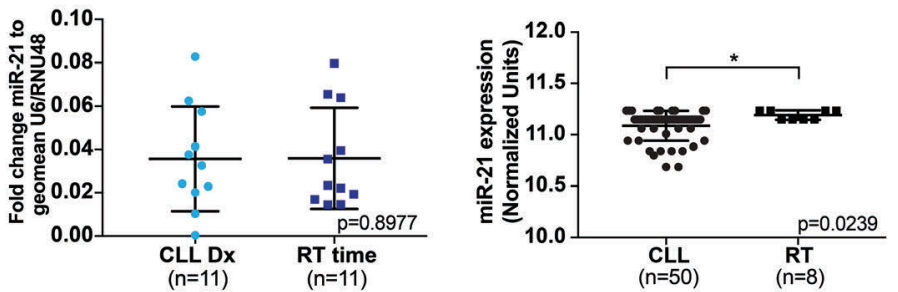

$p=0.9487$
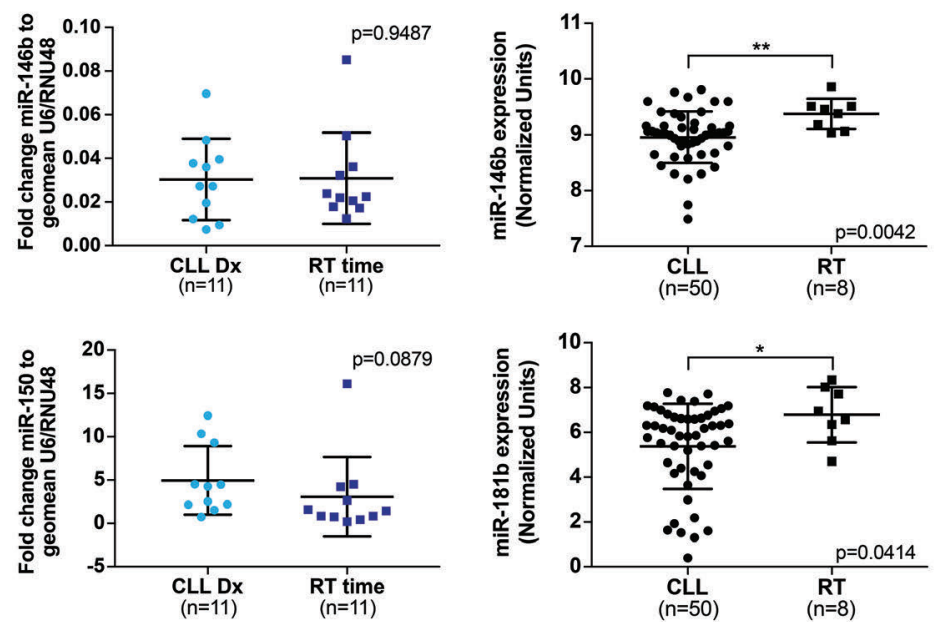

C

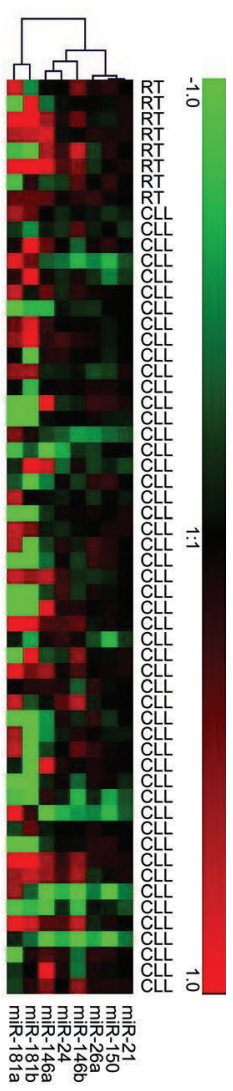

Figure 3. microRNA expression validation by quantitative reverse transcription polymerase chain reaction and gene expression profiling. (A) Quantitative reverse transcription polymerase chain reaction (qRT-PCR) expression analysis shows that miR-21 and miR-146b are significantly upregulated, and miR-150 is significantly downregulated at the time of Richter transformation (RT) when compared to their expression at the time of chronic lymphocytic leukemia (CLL) diagnosis (Dx) in the extended set of Richter syndrome (RS) samples, but not in the extended set of control CLL samples. (B) Gene expression profiling analysis in an independent set of RS/CLL samples from Ulm University shows significant upregulation of miR-21, miR-146b and miR-181b in RS when compared to CLL. (C) Heatmap showing the miRNA signature for CLL with subsequent transformation (“Richter") and CLL without transformation ("CLL") in the validation cohort ("UIm University") after hierarchical clustering on genes (Pearson correlation, average linkage). ${ }^{*} P<0.05 ; * * P<0.01$. For additional Information, see Online Supplementary Figure S3. 
The significantly differently expressed and hub-specific microRNA signature targets are enriched in pathways involved in cancer, immunity and inflammation

To understand the biological significance of the differentially expressed miRNA as well as of the RS-specific miRNA hubs from the above network analyses, we performed ingenuity pathway analysis for the validated targets (Online Supplementary Table $S 5 A, B$ ). Ingenuity pathway analysis of all targets of the four SDE miRNA of the restricted signature revealed the involvement of signaling pathways with a strong enrichment for pathways involved in cancer and also in immune responses (Figure 5C). We performed the same ingenuity pathway analysis on the three HUS Richter-specific miRNA (Figure 5D), and again found a strong enrichment towards cancer and autoimmune disorders/immunity-specific pathways. Interestingly, seven of the top 20 canonical pathways were common between the SDE miRNA and HUS
miRNA (Figure 5C,D). The connection with the p53 signaling pathway is compelling and can be considered as a "positive" control since this protein has a well-known role in the pathogenesis of RS, as well as aggressive CLL. ${ }^{14}$

\section{Functional characterization of the miRNA involved}

in Richter transformation

In order to start the functional characterization of the miRNA, we took advantage of a well characterized CLL xenograft model that employs primary CLL cells ${ }^{18-20}$ (Figure 6A). We checked the expression levels of the eight miRNA in the Richter transformation restricted and enlarged signatures. Since human CLL cells proliferate faster after transplantation into mice ${ }^{18-20,31}$ similar to what is observed after Richter transformation in humans, we hypothesized that this model partially resembles the functional phenotype observed in Richter transformation, characterized by higher proliferation rates compared to

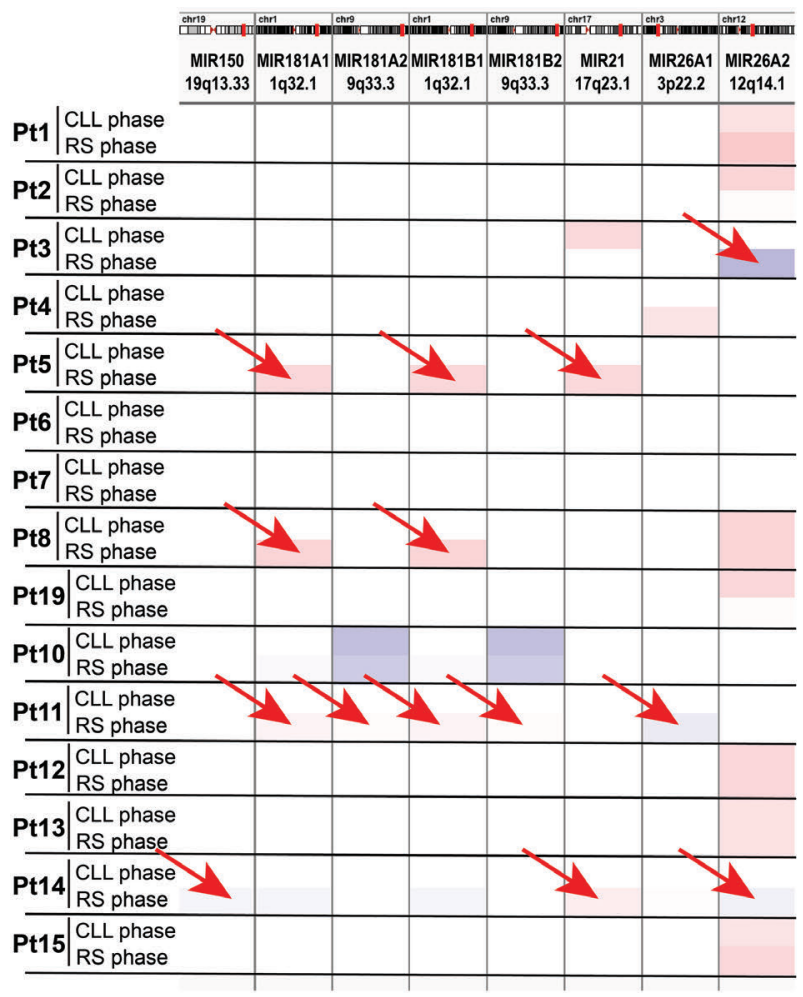

Figure 4. Genomic microRNA profiling in lymphoid tumors. (A) Analysis of miR-150, miR-181a/b, miR-21 and miR-26a genomic loci in a subset of 15 paired chronic lymphocytic leukemia (CLL) phase/Richter syndrome (RS) phase cases. (B) The genomic regions containing miR-21, miR-146a and miR-181a/b-1 are more often gained than lost in a set of 737 cases of mature lymphoid tumors. ${ }^{17,26-29}$ For additional Information, see Online Supplementary Figure S4.

B

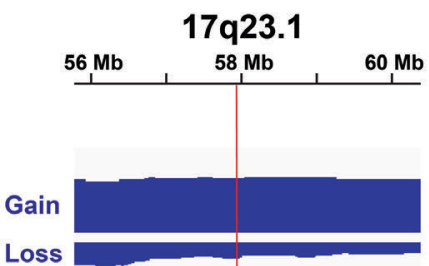

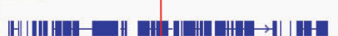

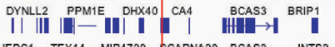
UEDC1 II TE14 MFA729 SCARNA20 BCAS3 INTS2
SRSF1 II TRIM37 TUED1
BZRAP1AS1 1
$5 q 33.3$

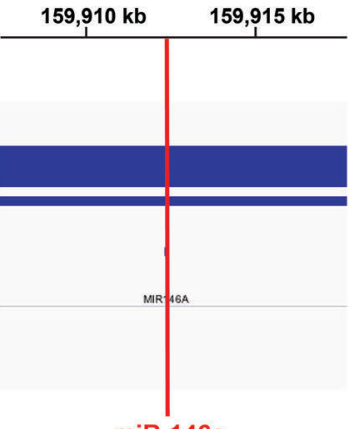

$\operatorname{miR}-146 a$
$1 q 32.1$

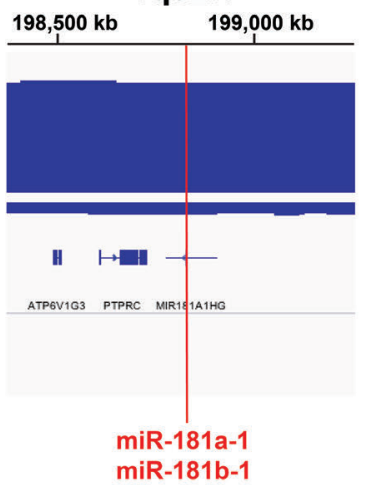




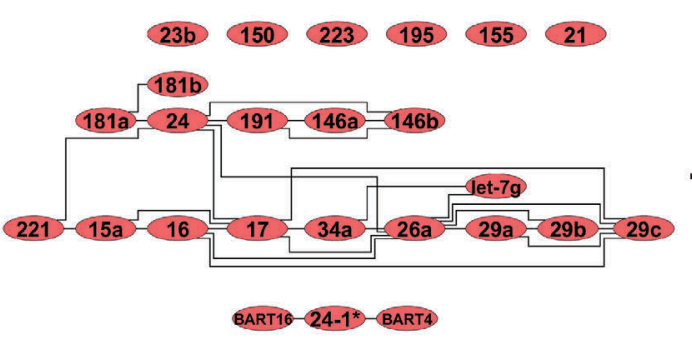

1a: Patients at the time of CLL diagnosis

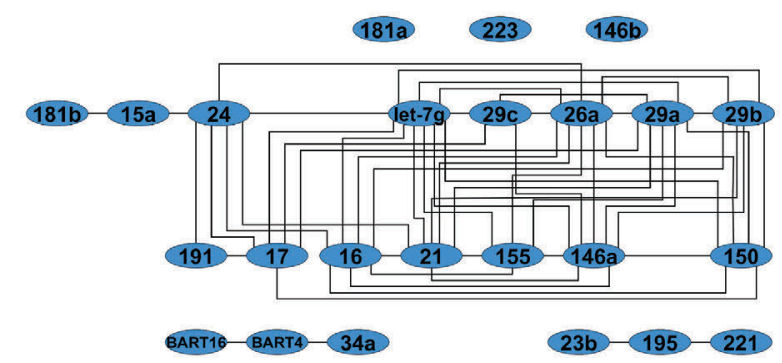

B

2a: Patients at the time of CLL diagnosis

PCR networks

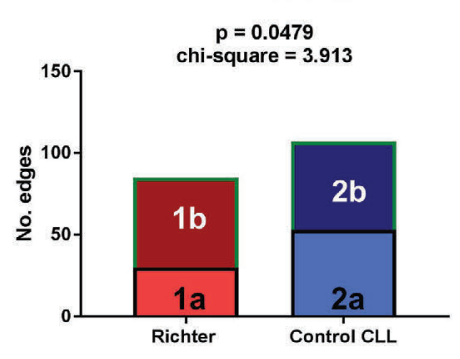

C

Canonical pathway analysis of "restricted signature" miRNAs confirmed targets tog(p-value)

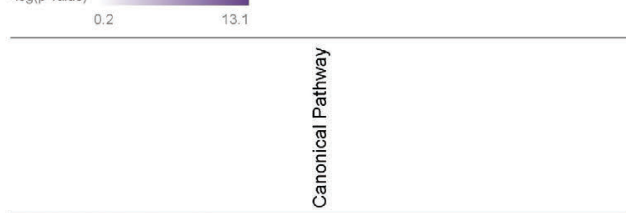

Molecular Mechanisms of Cancer
Neuroinflammation Signaling Pathway

NF-kB Signaling

NF-KB Signaling

Colorectal Cancer Metastasis Signaling

p53 Signaling

Osteoarthritis Pathway

Osteoarthritis Pa

Pancreatic Adenocarcinoma Signaling

Role of Macrophages, Fibroblasts and Endothelial Cells in Rheumatoid Arthritis

Aryl Hydrocarbon Receptor Signaling

HMGB1 Signalins

JAK/Stat Signaling

Hepatic Fibrosis / Hepatic Stellate Cell Activation

Glucocorticoid Receptor Signaling

Toll-like Receptor Signaling

IL-6 Signaling

Sumoylation Pathway

Th1 and Th2 Activation Pathway

PPAR $/$ RXRa Activatiol

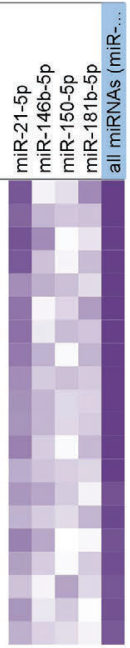

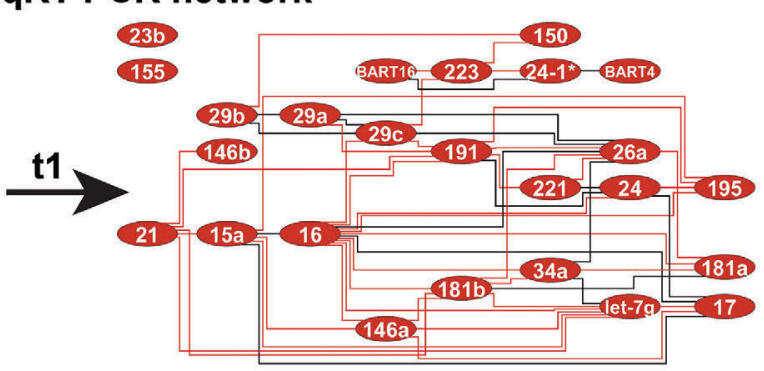

1b: Patients at the time of Richter transformation

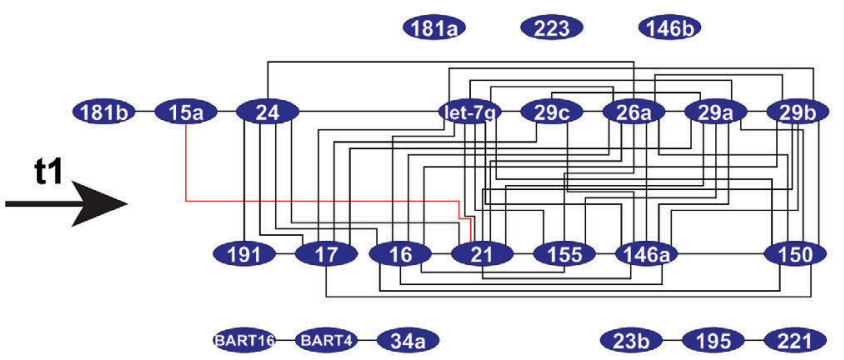

2b: CLL patients at the same follow-up time as RS

$1 \mathrm{a}$ vs $1 \mathrm{~b}$

$2 a$ vs $2 b$
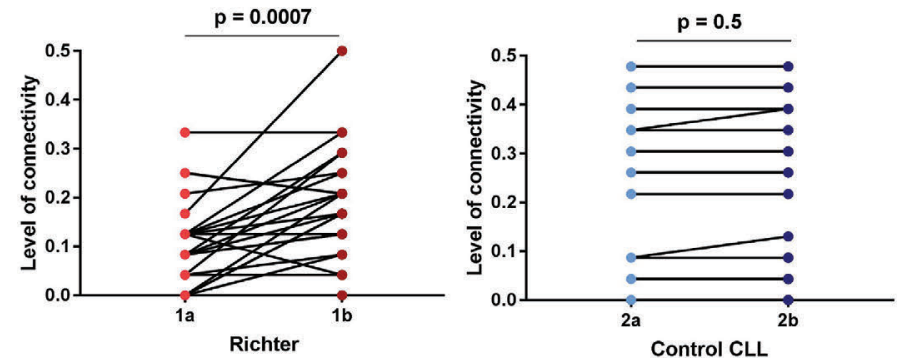

D

Canonical pathway analysis of miRNA hubs confirmed targets $\begin{array}{rl}-\log (p-v a l u e) \\ 0.2 & 18.8\end{array}$

Figure 5. microRNA network reprogramming during Richter transformation. (A) The 25-miRNA quantitative reverse transcription polymerase chain reaction (qRTPCR) expression network in the four groups analyzed. (B) Analysis of the number of edges (left panel) and connectivity (middle and right panels). (C) Ingenuity canonical pathway analysis - top 20 signaling pathways for validated targets of miR-21, miR-146b, miR-150 and miR-181b and (D) for the miRNA hubs specific for Richter transformation (miR-191, miR-17 and miR-29c). The heatmap shows the most significantly involved pathways, considering validated targets of all miRNA (last column). White to purple boxes represent $\log (P$-value) with the darkest boxes being the statistically most significant. $P$-values for targets of each miRNA separately are given in the first four columns. Due to the large number of targets, to increase the confidence of the data, we considered a $P<0.01$ as an inclusion criterion. For additional Information, see Online Supplementary Figure S5. 
A

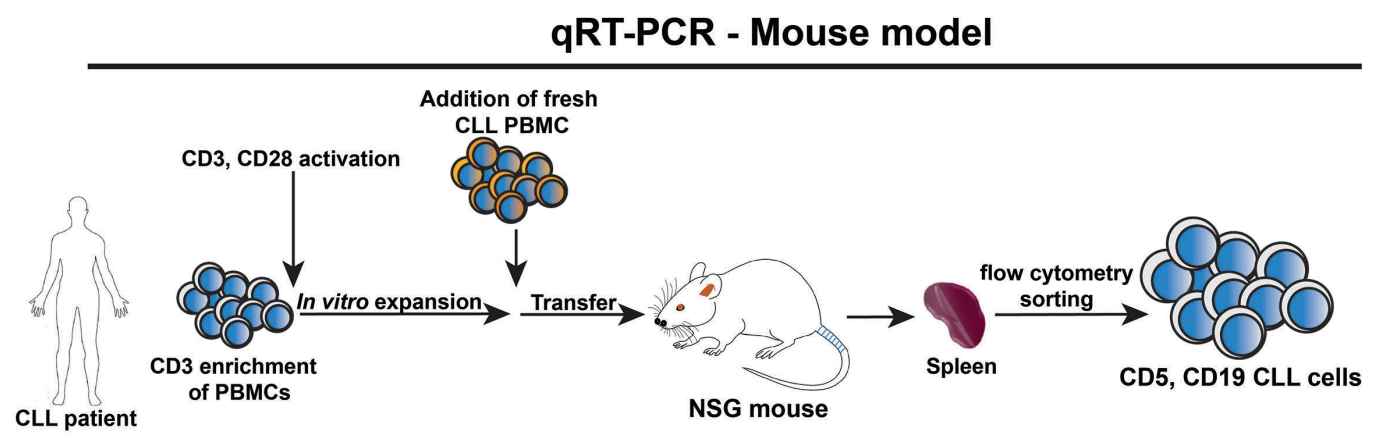

B
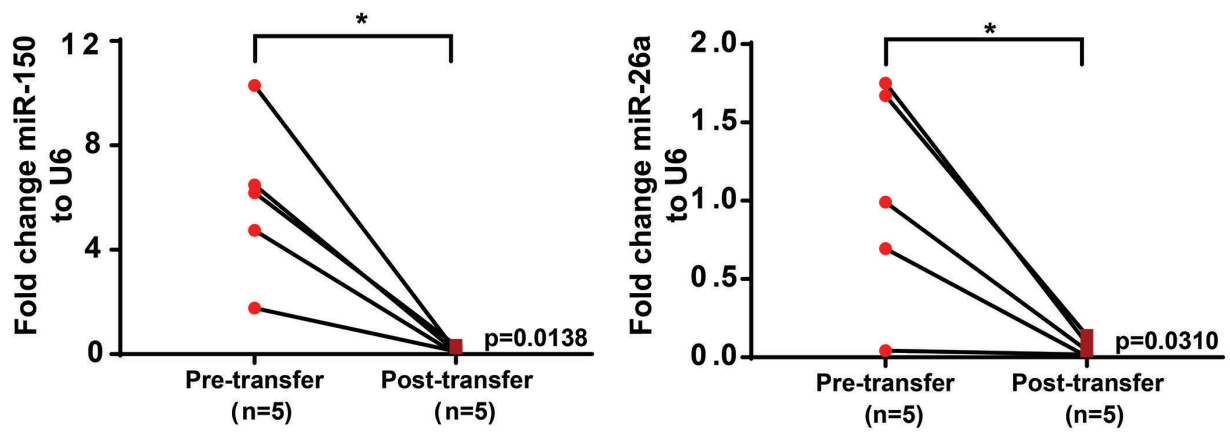

in vitro studies

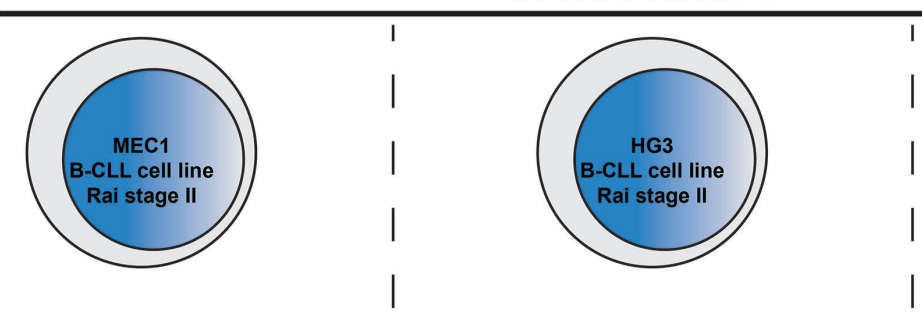

C
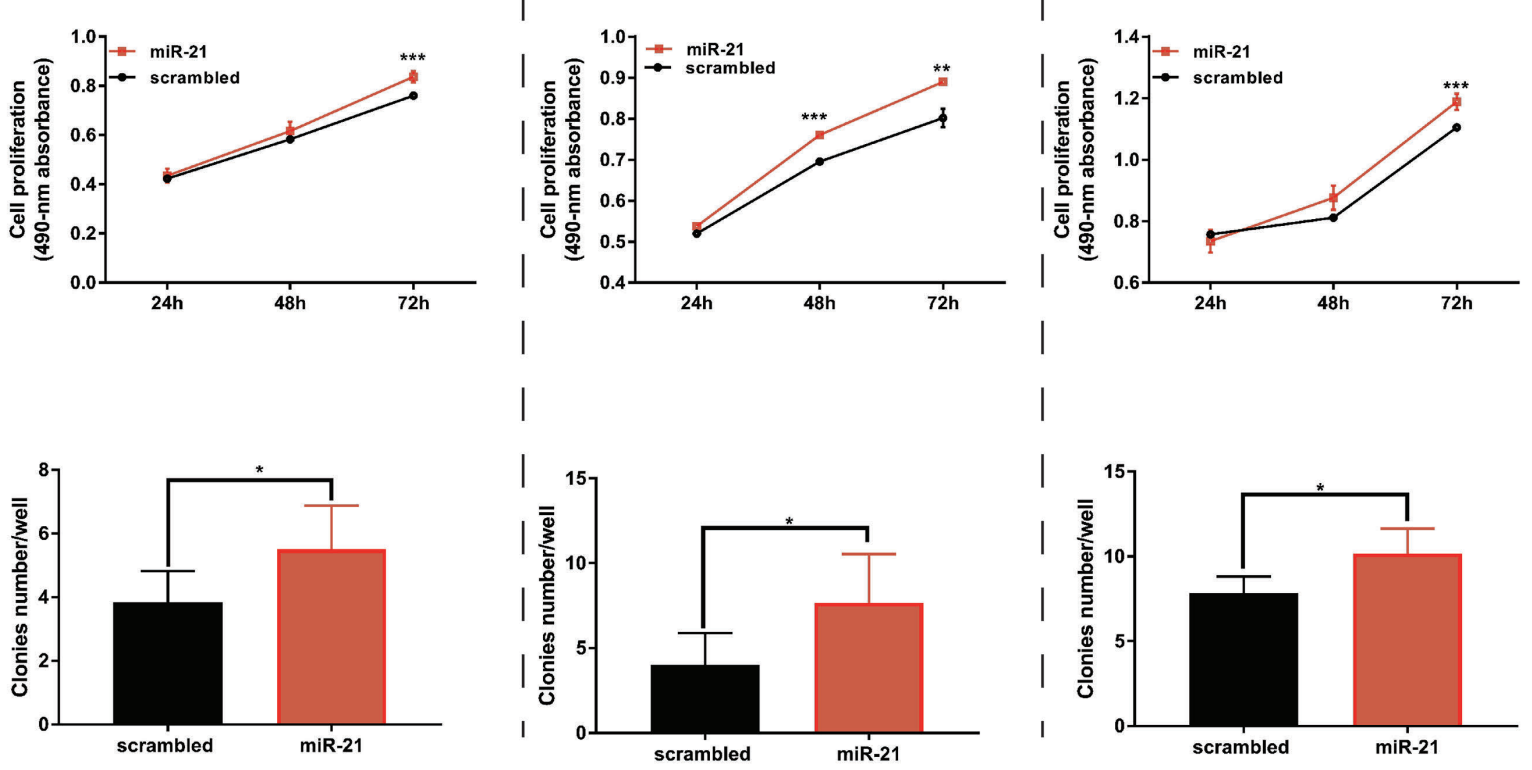

Figure 6. microRNA analysis after transfer of chronic lymphocytic leukemia B cells into NSG mice and in vitro functional studies. (A) Schematic representation of the chronic lymphocytic leukemia (CLL) mouse model. (B) miR-150 and miR-26a are significantly downregulated in post-transfer peripheral blood mononuclear cells in mice compared with pre-transfer CLL samples. (C) Two different CLL-specific cell lines - MEC1 and HG3, and one diffuse large B-cell lymphoma cell line - HB were used for functional assays (upper panel). Proliferation of MEC1, HG3 and HB cells was assessed by a CellTiter 96 AQueous One Solution assay. The cells were transfected with scrambled mimic or miR-21 mimic for 24, 48 and $72 \mathrm{~h}$ (middle panel). Representative images and graphs representing at least three independent experiments of the soft agar colony-formation assay for miR-21 mimic and control mimic transfected MEC1, HG3 and HB cells (lower panel). $* P<0.05$; $* * P<0.01$; $* * * P<0.001$. For additional Information, see Online Supplementary Figure S6. 
CLL. ${ }^{9}$ The levels of expression of miR-150 and miR-26a were found to be significantly downregulated in posttransfer samples compared with pre-transfer samples, independently of the IGHV mutated/unmutated status of the pre-transfer CLL samples (Figure 6B). This reduction was concordant with what we observed in the analyzed human cohorts (Figure 2A). Among the other six miRNA, we identified the same variation trends in miR-21 (2/5 cases) and miR-181b (3/5 cases) although statistical significance was not reached (Online Supplementary Figure 6A). Among canonical pathways common to both the miR150 and miR-26a validated targets, we identified mostly cancer-related pathways, including the TP53 pathway (Online Supplementary Figure 6B).

Since no RS cell lines have been reported before, while performing these studies, we used two different CLL cell lines (MEC1 and HG3) and one diffuse large B-cell lymphoma (DLBCL) cell line (HB) (Figure 6C) to investigate the role of miRNA from the "restricted signature" in cell proliferation and colony formation: only the ectopic overexpression of miR-21 through a miR-mimic significantly increased cell proliferation in all three cell lines, when compared with scrambled controls (Figure 6C). We further examined the long-term effects (up to 21 days) of miR-21 on CLL and DLBCL cell proliferation using a soft agar colony-formation assay. Overexpression of miR-21 significantly increased colony formation in all cell models when compared with scrambled controls (Figure 6C). There have been reports that aberrant expression of miR21 is associated with poor clinical outcome in patients with CLL..$^{32}$ These data support a functional effect of miR21 in both CLL and DLBCL cells that needs to be tested in future RS cell models.

\section{Discussion}

By using a stringent training/validation workflow on multiple, independent CLL/RS cohorts from several institutions, analyzed by three different expression profiling methods, we identified a miRNA signature that is involved in the process of transformation of CLL to RS. This signature is composed of the overexpressed miR-21, miR-146b and miR-181b, and by the downregulated miR150. These miRNA are SDE at the time of Richter transformation, but not after the same time of evolution of CLL in patients who never underwent Richter transformation ("control" group). miR-21 is a well-known oncogene, overexpressed and associated with poor prognosis in CLL, ${ }^{32,33}$ and highly expressed in patients who do not respond to fludarabine treatment. ${ }^{34}$ In addition, miR-21 transgenic mice spontaneously develop a pre-B-cell lymphoblastic lymphoma/leukemia. ${ }^{23}$ miR-150, on the other hand, is lowly expressed in CLL proliferation center ${ }^{35,36}$ and shows reduced expression in poor prognosis CLL. ${ }^{37}$ Recently, Balatti et al. reported that a signature of 23 miRNA is differentially expressed in CLL samples which developed RS compared with CLL samples which did not develop RS. In their study, it was demonstrated that miR$125 a-5 p$ is highly expressed, while miR-34a-5p is downregulated in pre-RS samples compared with samples from a control group and that deregulation of miR-34a and miR-125a-5p can predict $\sim 50 \%$ of RS with a false positive rate of $\sim 9 \%{ }^{38}$ The different results we obtained can be explained by the fact that we focused on miRNA which are deregulated in the stage of RS versus pre-RS (group $1 \mathrm{~b}$ versus group 1a) and not on miRNA that can predict Richter transformation (group 1a versus group 2a).

Of interest, the same four miRNA from this "restricted" signature are reported in literature as being differentially expressed in another fatal condition, sepsis (Online Supplementary Table S6), in the same way as in RS, making this signature similar to that of an acute infectious disease characterized by multiple organ failure. For example, tumor-secreted miR-21 can bind to toll-like receptors (TLR) on immune cells, triggering an inflammatory response that may contribute to tumor growth and metastasis. ${ }^{39,40}$ In addition, miR-21 suppresses T-cell apoptosis, ${ }^{41}$ and promotes Th17 cell differentiation, which is important for the development of multiple autoimmune diseases. ${ }^{42,43}$ miR-146b modulates the TLR4 signaling pathway through targeting of TLR4, MyD88, IRAK-1 and TRAF6, and has anti-inflammatory activity by reducing several pro-inflammatory cytokines, such as interleukin6, tumor necrosis factor- $\alpha$, interleukin-8 and CCL2/3/7.44,45 Furthermore, miR-150 is important for the development of mature natural killer cells, which are of primordial importance for the induction of adaptive immune responses, and defense against pathogens. ${ }^{46} \mathrm{miR}$ 150 can also decrease the production of inflammatory cytokines, such as interleukin-2 and tumor necrosis factor- $\alpha$, through disruption of CD28/B7 co-stimulatory signal transduction, resulting in immune tolerance. ${ }^{47}$ In the context of acute lung injury, miR-181b may stimulate inflammation through activation of nuclear factor- $\mathrm{kB},{ }^{48} \mathrm{a}$ pathway commonly deregulated in CLL, which is currently being investigated as a therapeutic target for the treatment of CLL and RS. ${ }^{49}$

To address our miRNA signature in the setting of markedly enhanced CLL B-cell proliferation, as occurs in patients with Richter transformation, we used a CLL primary xenograft mouse model and found that the levels of expression of miR-26a and miR-150 were significantly reduced in proliferating CLL cells purified from the spleen of xenografted mice compared to the levels in pre-transfer cells. miR-26a reduces the expansion/accumulation of leukemic cells of $\mathrm{E} \mu$-TCL1 mice and in vivo administration of miR-26a promotes apoptosis in mice..$^{50}$

Further support for this new view of the pathogenesis of RS, as a disease related to both malignant and immunerelated processes, comes from the network analysis we performed. miRNA networks in cancer have been described to be disjointed and composed of multiple subnetworks. ${ }^{51}$ In contrast, the miRNA network after Richter transformation (group 1b) contains a significantly higher number of edges, with almost all nodes connected to the main network.

A noteworthy observation is that the expression of viral miRNA, detected by the Firefly assay, shows a very high level of correlation, leading to the construction of a highly connected viral miRNA network. In all four networks (groups 1a, 1b, 2a, and 2b, see Online Supplementary Figure S5) the viral miRNA are all part of the main graph and miR-4286 and miR-1260a (a tRNA-derived miRNA) s2 $^{52}$ are always joining this main network. This observation leads us to the supposition that there is an unknown mechanism that controls the expression of the different viral miRNA simultaneously. Moreover, it has recently been shown in an in vivo model that immunosuppressive therapy leads to the reactivation of Epstein-Barr virus in CLL 
xenografts, increasing the risk of DLBCL (Richter transformation). ${ }^{53}$ The viral miRNA that correlate in our networks could be a marker of viral reactivation.

Several questions remain to be answered. First, what are the mechanisms that lead to the high level of correlation between miRNA during Richter transformation? Further investigation of the exact role of each of the signature miRNA and their mechanisms of action in the Richter transformation process is warranted. For this, the development of in vitro and in vivo models of CLL to RS transformation is imperative. Second, it was surprising that, out of 15 deregulated miRNA identified by the Firefly assay in the extended cohort, only three could be confirmed by qRT-PCR in the same dataset. This is most likely due to a combination of factors, such as the poor quality of RNA from FFPE samples, sensitivity of the assays, and the difficulty in identifying good normalizers. In the Firefly assay, a combination of miR-15a, miR-191 and miR-26a were found to be the best for normalization, while for the qRT-PCR experiments, the geometric mean of U6 and RNU48 was found to have the least variability. In addition, the Firefly platform is not based on PCR amplification, while qRT-PCR is, which may also partially explain the differences in results.

Some limitations need to be acknowledged with regard to this study. The number of patients included was small, so the statistical power of the analysis is limited. RS is a relatively rare disease and it is very difficult to obtain a large sample set. Furthermore, we analyzed only 40 human and viral miRNA using the Firefly assay and 25 miRNA using qRT-PCR, thus offering a limited view of the miRNA expression deregulation and miRNA network in RS. It is likely that other miRNA, which we overlooked by analyzing a restricted panel of miRNA, could be involved in the pathogenesis of RS. We tried to overcome this limitation by choosing the most suitable miRNA candidates and using previous RNA-sequencing data, ${ }^{20}$ and literature research of miRNA implicated in CLL.

Finally, the identification of SDE miR-21 (overexpressed) and miR-150 (downregulated) and of HUS miR$17,-29 \mathrm{c}$ and -191 demonstrates that RS is a genetically heterogeneous disease and offers new targets for the therapeutic development of anti-miRNA ${ }^{54}$ in this disease which currently does not have a confirmed treatment to increase the poor life expectancy.

\section{Acknowledgments}

We thank Mike Tackett from FirePlex Service at Abcam (Cambridge, MA, USA) for the technical support.

\section{Funding}

Dr. Calin is the Felix L. Haas Endowed Professor in Basic Science. Work in his laboratory is supported by National Institutes of Health (NIH/NCATS) grant UH3TR00943-01 through the NIH Common Fund, Office of Strategic Coordination (OSC), National Cancer Institute (NCI) grants 1R01 CA182905-01 and 1R01CA222007-01A1, an NIGMS 1R01GM122775-01 grant, a U54 grant - UPR/MDACC Partnership for Excellence in Cancer Research 2016 Pilot Project, a Team DOD (CA160445P1) grant, a Ladies Leukemia League grant, a CLL Moonshot Flagship project, a SINF 2017 grant, and the Estate of C. G. Johnson, Jr. Work by Dr. Van Roosbroeck was supported in part by the Lauri Strauss Leukemia Foundation. Dr. Dragomir was supported by POC grant n.35/01.09.2016, ID 37_796. Dr. Bertoni was supported by grants from the Helmut Horten Foundation, the San Salvatore Foundation, and by Oncosuisse (OCS - 02296-082008). Dr. Stilgenbauer and Dr. Bloehdorn were supported by the DFG through SFB1074 subprojects B1 and B2. Dr. You was supported in part by NIH/NCI RO1 CA164346 and IRG of the University of Texas MD Anderson Cancer Center. Dr. Gaidano was supported by AIRC $5 \times 1000$ project 21198, AIRC, Milan, Italy. Dr. Zupo was supported by Italian Ministry of Health $5 \times 1000$ funds 2014 and 2015 .

\section{References}

1. Ciccone M, Ferrajoli A, Keating MJ, Calin GA. SnapShot: chronic lymphocytic leukemia. Cancer Cell. 2014;26(5):770770.e1.

2. Kipps TJ, Stevenson FK, Wu CJ, et al. Chronic lymphocytic leukaemia. Nat Rev Dis Primers. 2017;3:16096.

3. Bullrich F, Fujii H, Calin G, et al. Characterization of the 13q14 tumor suppressor locus in CLL: identification of ALT1, an alternative splice variant of the LEU2 gene. Cancer Res. 2001;61(18):6640-6648.

4. Calin GA, Croce CM. Genomics of chronic lymphocytic leukemia microRNAs as new players with clinical significance. Semin Oncol. 2006;33(2):167-173.

5. Calin GA, Dumitru CD, Shimizu M, et al. Frequent deletions and down-regulation of micro- RNA genes miR15 and miR16 at 13 q14 in chronic lymphocytic leukemia. Proc Natl Acad Sci U S A. 2002;99(24): 15524-15529.

6. Aydin S, Rossi D, Bergui L, et al. CD38 gene polymorphism and chronic lymphocytic leukemia: a role in transformation to
Richter syndrome? Blood. 2008;111(12): 5646-5653.

7. Maddocks KJ, Ruppert AS, Lozanski G, et al. Etiology of ibrutinib therapy discontinuation and outcomes in patients with chronic lymphocytic leukemia. JAMA Oncol. 2015;1(1):80-87.

8. Roberts AW, Davids MS, Pagel JM, et al. Targeting BCL2 with venetoclax in relapsed chronic lymphocytic leukemia. N Engl J Med. 2016;374(4):311-322.

9. Rossi D, Gaidano G. Richter syndrome: pathogenesis and management. Semin Oncol. 2016;43(2):311-319.

10. Coiffier B, Lepage E, Briere J, et al. CHOP chemotherapy plus rituximab compared with CHOP alone in elderly patients with diffuse large-B-cell lymphoma. $N$ Engl J Med. 2002;346(4):235-242.

11. Dabaja BS, O'Brien SM, Kantarjian HM, et al. Fractionated cyclophosphamide, vincristine, liposomal daunorubicin (daunoXome), and dexamethasone (hyperCVXD) regimen in Richter's syndrome. Leuk Lymphoma. 2001;42(3):329337.

12. Tsimberidou AM, O'Brien SM, Cortes JE, et al. Phase II study of fludarabine, cytarabine (Ara-C), cyclophosphamide, cisplatin and GM-CSF (FACPGM) in patients with Richter's syndrome or refractory lymphoproliferative disorders. Leuk Lymphoma. 2002;43(4):767-772.

13. Tsimberidou AM, Wierda WG, Wen S, et al Phase I-II clinical trial of oxaliplatin, fludarabine, cytarabine, and rituximab therapy in aggressive relapsed/refractory chronic lymphocytic leukemia or Richter syndrome. Clin Lymphoma Myeloma Leuk. 2013;13 (5):568-574.

14. Rossi D, Spina V, Deambrogi C, et al. The genetics of Richter syndrome reveals disease heterogeneity and predicts survival after transformation. Blood. 2011;117(12): 3391-3401.

15. Falchi L, Keating MJ, Marom EM, et al Correlation between FDG/PET, histology, characteristics, and survival in 332 patients with chronic lymphoid leukemia. Blood. 2014;123(18):2783-2790.

16. Stilgenbauer S, Zenz T, Winkler D, et al. Subcutaneous alemtuzumab in fludarabinerefractory chronic lymphocytic leukemia: clinical results and prognostic marker analy- 
ses from the CLL2H study of the German Chronic Lymphocytic Leukemia Study Group. J Clin Oncol. 2009;27(24):39944001.

17. Chigrinova E, Rinaldi A, Kwee I, et al. Two main genetic pathways lead to the transformation of chronic lymphocytic leukemia to Richter syndrome. Blood. 2013;122(15): 2673-2682.

18. Bagnara D, Kaufman MS, Calissano C, et al. A novel adoptive transfer model of chronic lymphocytic leukemia suggests a key role for T lymphocytes in the disease. Blood. 2011;117(20):5463-5472.

19. Chen SS, Chiorazzi N. Murine genetically engineered and human xenograft models of chronic lymphocytic leukemia. Semin Hematol. 2014:51(3):188-205.

20. Patten PE, Ferrer G, Chen SS, et al. Chronic lymphocytic leukemia cells diversify and differentiate in vivo via a nonclassical Th1dependent, Bcl-6-deficient process. JCI Insight. 2016;1(4).

21. Ferrajoli A, Ivan $C$, Ciccone $M$, et al. Epstein-Barr virus microRNAs are expressed in patients with chronic lymphocytic leukemia and correlate with overall survival. EBioMedicine. 2015;2(6):572-582.

22. Vasilescu C, Dragomir M, Tanase M, et al. Circulating miRNAs in sepsis. A network under attack: an in-silico prediction of the potential existence of miRNA sponges in sepsis. PLoS One. 2017;12(8):e0183334.

23. Medina PP, Nolde M, Slack FJ. OncomiR addiction in an in vivo model of microRNA21-induced pre-B-cell lymphoma. Nature. 2010;467(7311):86-90

24. Calin GA, Sevignani C, Dumitru CD, et al. Human microRNA genes are frequently located at fragile sites and genomic regions involved in cancers. Proc Natl Acad Sci U S A. 2004:101(9):2999-3004.

25. Sevignani C, Calin GA, Nnadi SC, et al. MicroRNA genes are frequently located near mouse cancer susceptibility loci. Proc Natl Acad Sci U S A. 2007;104(19):80178022.

26. Boi M, Rinaldi A, Kwee I, et al. PRDM1/BLIMP1 is commonly inactivated in anaplastic large T-cell lymphoma. Blood. 2013;122(15):2683-2693

27. Martinez N, Almaraz C, Vaque JP, et al. Whole-exome sequencing in splenic marginal zone lymphoma reveals mutations in genes involved in marginal zone differentiation. Leukemia. 2014;28(6):1334-1340.

28. Rinaldi A, Kwee I, Young $\mathrm{KH}$ et al. Genome-wide high resolution DNA profiling of hairy cell leukaemia. Br J Haematol. 2013;162(4):566-569.
29. Rossi D, Trifonov V, Fangazio M, et al. The coding genome of splenic marginal zone lymphoma: activation of $\mathrm{NOTCH} 2$ and other pathways regulating marginal zone development. J Exp Med. 2012;209(9):15371551.

30. Van Roosbroeck K, Calin GA. MicroRNAs in chronic lymphocytic leukemia: miRacle or miRage for prognosis and targeted therapies? Semin Oncol. 2016;43(2):209-214.

31. Chiorazzi N. Cell proliferation and death: forgotten features of chronic lymphocytic leukemia B cells. Best Pract Res Clin Haematol. 2007;20(3):399-413.

32. Rossi S, Shimizu M, Barbarotto E, et al microRNA fingerprinting of CLL patients with chromosome $17 \mathrm{p}$ deletion identify a miR-21 score that stratifies early survival. Blood. 2010;116(6):945-952.

33. Fulci V, Chiaretti S, Goldoni $M$, et al. Quantitative technologies establish a novel microRNA profile of chronic lymphocytic leukemia. Blood. 2007;109(11):4944-4951.

34. Ferracin M, Zagatti B, Rizzotto L, et al. MicroRNAs involvement in fludarabine refractory chronic lymphocytic leukemia. Mol Cancer. 2010;9:123.

35. Szurian K, Csala I, Piurko V, et al. Quantitative miR analysis in chronic lymphocytic leukaemia/small lymphocytic lymphoma - proliferation centres are characterized by high miR-92a and miR-155 and low miR-150 expression. Leuk Res. 2017;58:39-42

36. Wang M, Tan LP, Dijkstra MK, et al. miRNA analysis in B-cell chronic lymphocytic leukaemia: proliferation centres characterized by low miR-150 and high BIC/miR-155 expression. J Pathol. 2008;215(1):13-20.

37. Mraz M, Chen L, Rassenti LZ, et al. miR 150 influences B-cell receptor signaling in chronic lymphocytic leukemia by regulating expression of GAB1 and FOXP1. Blood. 2014;124(1):84-95.

38. Balatti V, Tomasello L, Rassenti LZ, et al MiR-125a and MiR-34a expression predicts Richter syndrome in chronic lymphocytic leukemia patients. Blood. 2018;132(20): 2179-2182.

39. Bayraktar R, Van Roosbroeck K, Calin GA. Cell-to-cell communication: microRNAs as hormones. Mol Oncol. 2017;11(12):16731686.

40. Fabbri $M$, Paone A, Calore $F$, et al. MicroRNAs bind to Toll-like receptors to induce prometastatic inflammatory response. Proc Natl Acad Sci U S A. 2012; 109(31):E2110-2116.

41. Meisgen F, Xu N, Wei T, et al. MiR-21 is upregulated in psoriasis and suppresses $\mathrm{T}$ cell apoptosis. Exp Dermatol. 2012;21(4):312 314

42. Wang S, Wan X, Ruan O. The microRNA 21 in autoimmune diseases. Int J Mol Sci. 2016;17(6).

43. Xu WD, Pan F, Li JH \& Ye DO. MicroRNA21 with therapeutic potential in autoimmune diseases. Expert Opin Ther Targets. 2013;17(6):659-665.

44. Curtale G, Mirolo M, Renzi TA, et al Negative regulation of Toll-like receptor 4 signaling by IL-10-dependent microRNA146b. Proc Natl Acad Sci U S A 2013;110(28):11499-11504.

45. Taganov KD, Boldin MP, Chang KJ Baltimore D. NF-kappaB-dependent induction of microRNA miR-146, an inhibitor targeted to signaling proteins of innate immune responses. Proc Natl Acad Sci U S A. 2006;103(33):12481-12486.

46. Bezman NA, Chakraborty $\mathrm{T}$, Bender $\mathrm{T}$, Lanier L L. miR-150 regulates the development of NK and iNKT cells. J Exp Med. 2011;208(13):2717-2731.

47. Sang W, Wang Y, Zhang C, et al. MiR-150 impairs inflammatory cytokine production by targeting ARRB-2 after blocking CD28/B7 costimulatory pathway. Immunol Lett. 2016;172:1-10.

48. Wang Y, Mao G, Lv Y, Huang Q, Wang G. MicroRNA-181b stimulates inflammation via the nuclear factor-kappaB signaling pathway in vitro. Exp Ther Med 2015;10(4): 1584-1590.

49. Vaisitti T, Gaudino F, Ouk S, et al. Targeting metabolism and survival in chronic lymphocytic leukemia and Richter syndrome cells by a novel NF-kappaB inhibitor Haematologica. 2017;102(11):1878-1889.

50. D'Abundo L, Callegari E, Bresin A, et al Anti-leukemic activity of microRNA-26a in a chronic lymphocytic leukemia mouse model. Oncogene. 2017;36(47):6617-6626.

51. Volinia S, Galasso M, Costinean S, et al. Reprogramming of miRNA networks in cancer and leukemia. Genome Res. 2010;20(5): 589-599.

52. Venkatesh T, Suresh PS, Tsutsumi R. tRFs miRNAs in disguise. Gene. 2016;579(2):133 138.

53. Garcia-Barchino MJ, Sarasquete ME, Panizo C, et al. Richter transformation driven by Epstein-Barr virus reactivation during therapy-related immunosuppression in chronic lymphocytic leukaemia. J Pathol. 2018;245 (1):61-73.

54. Shah MY, Ferrajoli A, Sood AK, LopezBerestein G, Calin GA. microRNA Therapeutics in cancer - an emerging concept. EBioMedicine. 2016;12:34-42. 